\title{
Lung cancer, elderly and immune checkpoint inhibitors
}

\author{
Francesca Casaluce ${ }^{1}$, Assunta Sgambato ${ }^{1}$, Paolo Maione ${ }^{1}$, Alessia Spagnuolo ${ }^{1,2}$, Cesare Gridelli ${ }^{1}$ \\ ${ }^{1}$ Division of Medical Oncology, 'S. G. Moscati' Hospital, Avellino, Italy; ${ }^{2}$ Department of Clinical and Experimental Medicine, Second University of \\ Naples, Naples, Italy \\ Contributions: (I) Conception and design: All authors; (II) Administrative support: All authors; (III) Provision of study materials or patients: All \\ authors; (IV) Collection and assembly of data: All authors; (V) Data analysis and interpretation: All authors; (VI) Manuscript writing: All authors; (VII) \\ Final approval of manuscript: All authors. \\ Correspondence to: Cesare Gridelli, MD. Division of Medical Oncology, 'S. G. Moscati’ Hospital, Avellino, Italy. Email: cgridelli@libero.it.
}

\begin{abstract}
Lung cancer is predominantly a disease of the elderly with about $50 \%$ of diagnoses in patients aged $\geq 70$ years and about $14 \%$ in those older than 80 . Medical and physiological characteristics of elderly cancer patients make the choice of their better treatment more challenging. Furthermore, aging is accompanied by the so called "immunosenescence" phenomenon, the age-related decline in the immune system that is one of the potential reasons of increase of the incidence and prevalence of most cancers. There is a growing interest in understanding of immunosenescence and how it may correlate with the use of immune checkpoint inhibitors in elderly non-small cell lung cancer (NSCLC) patients. The survival benefit achieved by immunotherapy in all histologies and therapy line settings, added to its manageable toxicity profile, has dramatically changed the scenario of advanced NSCLC treatment. At subgroup analyses of randomized clinical trials, elderly NSCLC population seems to benefit from anti-programmed death-1 (anti-PD-1)/anti-programmed death ligand-1 (anti-PD-L1) agents' treatment. These efficacy data were also confirmed by studies in real-life setting. The key-points of aging and immunosenescence are described, focusing on the role of immune checkpoint inhibitors in elderly NSCLC population.
\end{abstract}

Keywords: Anti-programmed death-1 agents (anti-PD-1 agents); anti-programmed death ligand-1 agents (antiPD-L1 agents); elderly; immunosenescence; immune checkpoint inhibitors; non-small cell lung cancer

Submitted Feb 08, 2018. Accepted for publication May 10, 2018.

doi: $10.21037 /$ jtd.2018.05.90

View this article at: http://dx.doi.org/10.21037/jtd.2018.05.90

\section{Introduction}

Lung cancer, distinct in small (SCLC) and non-small [(NSCLC), about $85 \%$ of lung cancers] cell lung cancer, is predominantly a disease of the elderly with about $50 \%$ of diagnoses in patients aged $\geq 70$ years and about $14 \%$ in those older than 80 (1). Medical and physiological characteristics of elderly cancer patients make the choice of their better treatment more challenging. Additionally, these patients generally are excluded from clinical trials purely on the basis of chronological age, leading to their under-representation in clinical research.

Furthermore, physiological changes in body composition (such as increased body fat, reduced total body water) and progressive organ functional reserve failure (mainly of kidney, liver, hearth and bone-marrow) related to aging lead to elderly poor tolerance to stress, such as chemotherapy (2).

The cut-off of age for defining a patient "elderly" remains an open question because the physiological age is poorly reflected in chronological age. In clinical practice, the first one instead of the second one should be considered.

Unfortunately, to date laboratory tests and geriatric evaluations are inadequate to define aging, therefore chronological age remains the reference for clinical trials: the most suitable cut-off seems to be age 70 due to the higher prevalence of aging-related changes over 70 years (2).

In general, the management of the older person with cancer should be based on two key points: risk/benefit assessment and institution of multidisciplinary interventions (medical, psychological and social) that may improve the 
tolerance of chemotherapy by individual patient (2).

Furthermore, the comprehensive geriatric assessment (CGA), a multidimensional, interdisciplinary diagnostic process including the evaluation of medical, psychological and functional capabilities of a frail elderly person, should be used in order to plan and individualize the medical treatment $(2,3)$. This assessment, taken together with the evaluation of toxicity profile of each drug, have to guide the choice of the best treatment $(2,3)$.

Another important aspect of aging is the so called "immunosenescence" phenomenon, the age-related decline in the immune system, that is one of the potential reasons of increase of the incidence and prevalence of most cancers with age. There is a growing interest in immunosenescence and how it may correlate with the use of immune checkpoint inhibitors in elderly NSCLC patients $(4,5)$. Indeed, a paradoxical stimulation of tumor growth on institution of treatment with checkpoint inhibitors, probably because of altered immune function due to immunosenescence, has been reported in a recent retrospective analysis up to $10 \%$ of patients—mostly elderly, aged over 65 (6).

\section{The immune system and immunosenescence phenomenon}

The immune system is organized into innate and adaptive categories. The first provides an immediate defense against insult or infection involving physical epithelial barriers, phagocytes, natural killer (NK) cells, and circulating complement proteins. The adaptive immune response is initiated when tumor cell antigens released by innate immunity are taken up by antigen presenting cells (APCs), such as dendritic cells, that internalize and process tumor antigen onto major histocompatibility complex (MHC) molecules, which are expressed on the surface of APCs. This process triggers expression of $\mathrm{B} 7-1$ and $\mathrm{B} 7-2$ on the APCs, which migrate to lymph nodes. In the lymph node, the APCs present tumor antigens to naïve $T$ cells though interaction with the antigen-specific $\mathrm{T}$ cell receptor resulting in the priming and activation of effector $\mathrm{T}$ cell responses. In addition to antigen presentation in the context of an MHC molecule, T-cell activation requires the costimulatory signal stimulation by interaction between B7-1 or B7-2 on APC and CD28 on the T-cell. The major subtypes of $\mathrm{T}$ cells are $\mathrm{CD} 4^{+}$helper $\mathrm{T}$ cells (facilitating antibody production), $\mathrm{CD} 8^{+}$cytotoxic $\mathrm{T}$ cells (directly killing cells recognized as foreign) and regulatory $\mathrm{T}$ cells which have a role in modulating immune response. At this stage, the critical balance representing the ratio of $\mathrm{T}$ effector cells versus $\mathrm{T}$ regulatory cells determines the nature of the immune response. Then, the activated effector $T$ cells traffic to and infiltrate the tumor bed, recognize and binds the cancer cells by the interaction between its $\mathrm{T}$ cell receptor and its cognate antigen expressed on the tumor in association with MHC. Finally, they release cytolytic enzymes and cytokines, which recruit other members of the immune system, and proliferates (7).

However, the immune responses can damage healthy tissue if left unchecked. Immune checkpoint pathways, such as cytotoxic T-lymphocyte-associated antigen-4 (CTLA-4) and programmed cell death protein-1 (PD-1) pathways, are some of several mechanisms existing to downregulate immune responses. These receptors are similar in structure and are both expressed on T cells (PD-1 can be expressed also on B cells or NK cells), but they bind distinct ligands.

CTLA-4 primarily regulates early $\mathrm{T}$ cell activity and is upregulated on T cells after exposure to antigen. CTLA-4 competes for binding ligands B7-1 and B7-2 on the surface of APCs with much higher affinity than CD28, preventing these ligands from interaction to CD28 and blocking intracellular signaling that leads to T-cell proliferation (8).

While CTLA-4 functions largely in lymphoid organs and acts in the early stages of an immune response, PD-1 inhibition occurs primarily at the site of the tumor and functions in the later stages. PD-1 is a negative regulator of T-cell activity that inhibits kinase signaling pathwaysnormally leading to T-cell activation-through interaction with its two ligands PD-1/PD-L1, expressed both on APCs or tumor cells. This binding reduces T-cell proliferation, alters cytokine production and induces $\mathrm{T}$ cells to exhaustion and/or apoptosis (8). The PD-1 pathway is important in the tumor microenvironment, where PD-L1 expressed by tumors interacts with PD-1 on T cells to suppress T-cell effector function.

The immunosenescence includes reductions in B- and T-cell proliferation and function, quantitative differences in cellular subsets, functional impairment, and qualitative changes in APCs and an accumulation of regulatory $\mathrm{T}$ cells. The most pronounced age-associated changes occur in $\mathrm{T}$ cell composition. In the elderly, the functional decrease in naive $\mathrm{T}$ cell, due to thymic involution, is accompanied by a progressive conversion of naïve $T$ cells into virtual memory cells, due to chronic antigenic stimulation. These changes lead to reduced antigenic diversity of immune cells potentially associated with detrimental immune response to pathogens and tumor cells (5). 
Table 1 Main phase III clinical trials in NSCLC in the elderly

\begin{tabular}{|c|c|c|c|c|c|c|}
\hline Agent & Trial & Setting & $\begin{array}{l}\text { Median age ICls } \\
\text { treated year [range] }\end{array}$ & $\begin{array}{l}\text { Elderly patients ICls } \\
\text { treated [age ranges] }\end{array}$ & OS HR $(95 \% \mathrm{Cl})$ & $\begin{array}{l}\text { PFS HR } \\
(95 \% \mathrm{Cl})\end{array}$ \\
\hline \multirow[t]{3}{*}{ Nivolumab } & CheckMate 017 & Pretreated squamous & 62 [39-85] & 91 [65-75] & $0.56(0.34-0.91)$ & $0.51(0.32-0.82)$ \\
\hline & CheckMate 057 & Pretreated non-squamous & $61[37-84]$ & 200 [65-75] & $0.63(0.45-0.89)$ & $0.94(0.69-1.27)$ \\
\hline & & & & $43[\geq 75]$ & $0.90(0.43-1.87)$ & $0.97(0.49-1.95)$ \\
\hline \multirow[t]{2}{*}{ Pembrolizumab } & Keynote-010 & $\begin{array}{l}\text { Pretreated squamous and } \\
\text { non-squamous PD-L1 } \geq 1 \%\end{array}$ & 63 [56-69] & $429[\geq 65]$ & $0.76(0.57-1.02)$ & $0.93(0.72-1.19)$ \\
\hline & Keynote-024 & $\begin{array}{l}\text { First-line squamous and non- } \\
\text { squamous PD-L1 } \geq 50 \%\end{array}$ & 64.5 [33-90] & $164[\geq 65]$ & NR & $0.45(0.29-0.70)$ \\
\hline
\end{tabular}

$\mathrm{Cl}$, confidence interval; HR, hazard ratio; ICls, immune checkpoint inhibitors; NR, not reported; OS, overall survival; PD-L1, programmed death ligand; PFS, progression-free survival.

Concurrently, the lower capacity to neutralize newly discovered antigens with aging resulted from the reduction of naive $\mathrm{CD}^{+}$and the increasement of $\mathrm{CD}^{+}$and $\mathrm{CD} 8^{+}$ antigen experienced $\mathrm{T}$ cells. Furthermore, decrease of costimulatory (like CD28 and CD27 on T lymphocytes) and apoptotic molecules (like CD95) were described in the elderly (5). Regarding B lymphocytes, the senescence leads to a faster age-related decline of B cells number in the peripheral blood, in comparison with any other cell type, and to a paradoxically increase of immunoglobulins level. Furthermore, the decrease of antibodies with high antigen specificity and affinity and the increase of autoantibodies and proinflammatory cytokines leads to chronic state of low grade inflammation, known as "inflammaging".

Immunosenescence phenomenon involves also innate immune response affecting negatively different functions of APC (such as diminished antigen processing capacity through age-related injury of proteasome functions), reducing secretion of proinflammatory cytokines and lack of expression of toll-like receptors (5). These are only the main characteristics of the complex process of immunosenescence.

\section{Immune checkpoint inhibitors in NSCLC: focus on elderly patients}

As explained earlier, immune checkpoints are a mechanism of T-cell immune response modulation at APC-T-cell interface (priming phase) and at tumor microenvironment (effector phase). Several immune checkpoint inhibitors acting against $\mathrm{PD}-1 / \mathrm{PD}-\mathrm{L} 1$ and CTLA-4 have been approved in multiple cancers. However, limited efficacy and safety data are available in subgroup of elderly patients due to low accrual of 65 years or older aged patients and even lower of 75 years or older one in clinical trials. To date, no randomized phase III trials on efficacy of PD-1/PD-L1 targeting agents in the elderly lung patients with advanced NSCLC are available: data have been extrapolated from each key phase III trial (Table 1).

\section{Anti-PD-1 agents: nivolumab and pembrolizumab}

Nivolumab is a fully human IgG4 PD-1 immune-checkpoint inhibitor antibody that disrupts PD-1-mediated signaling and restores antitumor immunity, approved for pretreated advanced NSCLC patients, regardless PD-L1 expression level. Nivolumab has been tested as monotherapy in 129 heavily pretreated cancer in a phase I trial (CheckMate 003) $(9,10)$, in 117 squamous patients in a single-arm phase II trial (CheckMate 063) $(11,12)$, in second-line in 272 squamous and 582 non-squamous NSCLC compared to docetaxel in two randomized phase III trials (CheckMate 017 and 057 , respectively) (13-15) and in first-line versus platinum-based chemotherapy in 541 patients with PD-L1 of $1 \%$ or more in phase III trial (CheckMate 026) (16).

Long-term follow-up is available from the earlier phase I trial in 129 heavily pretreated patients (54\% pretreated at least three prior systemic therapies) enrolled regardless of 
their PD-L1 status and randomly assigned to three different dose levels of nivolumab (1, 3, or $10 \mathrm{mg} / \mathrm{kg}$ every 2 weeks). At 5 years, the estimated overall survival (OS) rate for all doses was $16 \%$ (consistently across histologies: $16 \%$ in squamous and $15 \%$ in non-squamous), and median OS was 9.9 months. In 69 patients with evaluable PD-L1 expression (53\% of patients), 5 -year OS rates increased as the PD-L1 expression level increased: $20 \%, 23 \%$ and $43 \%$ in patients with PD-L1 expression $<1 \%, \geq 1 \%$ and $\geq 50 \%$, respectively. Among 61 patients with unknown PD-L1 expression levels ( $47 \%$ of patients), the estimated 5 -year OS rate was $10 \%$ (9). In thirty-nine patients aged more than 70 years $(30 \%$ of population), the ORR (17.9\%) was overlapping to overall population and younger patients (10).

Of a total of 117 squamous patients enrolled in CheckMate 063,59 patients (50\% of population) were older than 65 years: an ORR of $14 \%$ was reported in overall population, $17 \%$ and $12 \%$ in those patients aged $\geq 65$ and $<65$, respectively (11). At 2-year follow-up, the 12 - and 18 -month OS rates were $39 \%$ and $27 \%$, respectively, and median OS was 8.1 months in overall population enrolled. The ORR was $15 \%$ (12).

In both squamous and non-squamous population $(13,14)$, nivolumab as second-line treatment reported a significantly improvement in OS, with the risk of death $41 \%$ and $27 \%$ lower than docetaxel (HR 0.59, $\mathrm{P}<0.001$ in CheckMate 017; HR 0.73, $\mathrm{P}=0.002$ in CheckMate 057), a longer median survival (3.2 and 2.8 months, respectively), and higher 1-year survival rate (1-year OS rate $42 \%$ vs. $24 \%$ in CheckMate 017 and $51 \%$ vs. $39 \%$ in CheckMate 057$)(13,14)$. While in squamous population PD-L1 expression was neither prognostic nor predictive of benefit, in non-squamous patients nivolumab was associated with even greater efficacy in subgroups defined according to prespecified levels of PD-L1 expression $(\geq 1 \%, \geq 5 \%$, and $\geq 10 \%)$.

After a minimum follow-up of 36.6 months in each study, nivolumab confirmed the OS benefit versus docetaxel (3year OS rates: $16 \%$ versus $6 \%$ in CheckMate 017 and $18 \%$ versus $9 \%$ in CheckMate 057 , respectively). Consistent with previously data, while the OS benefit was reported regardless of PD-L1 expression in squamous NSCLC, a higher PD-L1 expression levels were associated with greater OS benefit in non-squamous NSCLC (15).

Focusing on survival outcomes in pre-defined aged subgroups, 91 patients were aged 65 to 75 years $(33 \%$ of population) and 29 patients were older than 75 years (10\%) in CheckMate 017. Nivolumab achieved a reduction of $49 \%$ of the risk of death in the $65-75$ aged group (HR 0.56 ,
95\% CI, 0.32-0.82), while a no significant HR for survival was observed in patients aged $\geq 75$ years (HR $1.85,95 \%$ CI, 0.76-4.51) (13). Similar to squamous population, in CheckMate 057 trial a $37 \%$ reduction of risk of death was reported in 200 patients (34\% of population) aged $65-75$ years (HR 0.63, 95\% CI, 0.45-0.89), while a no significant $10 \%$ reduction of death in 43 patients $(7 \%)$ aged $\geq 75$ years (HR 0.90; 95 \% CI, 0.43-1.87) (14). The non-significant HR for survival observed in patients aged $\geq 75$ years in both histologies might be attributable to small sample size and type 1 error for multiple comparisons.

Fatigue (16\% each one), decreased appetite (10\% and $12 \%$, respectively), asthenia (10\% each one), and nausea ( $9 \%$ and $12 \%)$ were the most frequently reported treatmentrelated adverse events (AEs) in CheckMate 017 and 057 trials, respectively $(13,14)$. Among the most frequently immuno-related AEs were any grade hypothyroidism (4\% and $7 \%$ ), diarrhea ( $8 \%$ each one), pneumonitis (5\% and $3 \%)$, rash (4\% and $9 \%)$, increased alanine and aspartate aminotransferase levels in squamous and non-squamous populations, respectively $(13,14)$. No significant difference was noted among all age groups was reported from a pooled analysis of two studies, CheckMate 063 and 017 (17).

CheckMate 153 is an ongoing, predominantly community-based, phase IIIB/IV safety study of nivolumab in pretreated advanced NSCLC patients in the United States/Canada. Of 1,308 patients, 520 (40\%) were aged $\geq 70$ years and their estimated 6-month OS $(63 \%$; $95 \%$ CI, $58-67 \%)$ was comparable with that for patients aged $<70$ years $(63 \%$; 95\% CI, 59-67\%). Likewise, nivolumab safety profile was similar in the two aged subgroups (any grade AEs: 62\% vs. 59\%; grade 3-4 AEs: $12 \%$ vs. $11 \%$; grade $5 \mathrm{AEs}:<1 \%$ each one, in age $<70$ and $\geq 70$ years, respectively) (18).

A retrospective cohort study of 173 advanced NSCLC, including 43 patients $\geq 75$ years old, treated with nivolumab outside of clinical trials, confirmed that elderly patients gained similar benefit from nivolumab compared to younger patients reporting no differences in ORR (OR 1.0; $\mathrm{P}=0.97)$, progression-free survival (PFS), (HR 0.71; $\mathrm{P}=0.12$ ), or OS (HR 0.8; $\mathrm{P}=0.4)(19)$. Confirmatory data came from expanded access program (EAP) conducted in Italy in both histologies in second or more line of treatment $(20,21)$. Regarding squamous histology, of a total of 372 patients, $70(18.8 \%)$ were $\geq 75$ years old. On the other hand, of 1,588 non-squamous NSCLC patients, $522(33 \%)$ were $\geq 70$ years and $232(15 \%)$ were $\geq 75$ years. In both histologies, nivolumab treatment confirmed its efficacy, reporting 
a median PFS and median OS of 3.2 and 7.6 months, respectively, in squamous population, while a median OS of 11.5 and 12.0 months in non-squamous patient aged $\geq 70$ and $\geq 75$, respectively. Safety results were in line with what previously reported, with discontinuation due to related AEs occurring in only $8(11.4 \%)$ squamous patients, 25 $(5 \%)$ non-squamous patients aged $>70$, and $13(6 \%)$ nonsquamous patient aged $\geq 75(20,21)$.

Concerning first-line, in CheckMate 026 study nivolumab was not associated with significantly longer PFS than chemotherapy among $423 / 540$ patients with a PD-L1 expression level of $5 \%$ or more (4.2 vs. 5.9 months with chemotherapy, HR $1.15 ; \mathrm{P}=0.25)$. A similar OS was observed between the groups (14.4 vs. 13.2 months; HR 1.02) (16). Among 423 patients with PD-L1 >5\%, 196 (about $43 \%$ of the population) were over 65 years. In both aged subgroups ( $<65$ and $\geq 65$ years), nivolumab performed equivalently to platinum-based chemotherapy in terms of both PFS and OS (in aged $\geq 65$ years, median PFS 4.2 vs. 5.7 months with chemotherapy, HR 1.30; median OS $14.7 v s$. 11 months with chemotherapy, HR 0.98) (16).

Pembrolizumab is a fully humanized IgG4 antibody against the PD-1 receptor, approved for PD-L1 positive advanced NSCLC patients, specifically as first-line for PD-L1 TPS $\geq 50 \%$ and as second-line or more for PDL1 al least 1\%. In the phase II/III Keynote-010 trial, pembrolizumab improved OS in pretreated patients with PD-L1 positive (PD-L1 expression level over than 1\%) advanced NSCLC. Compared to docetaxel, both doses of pembrolizumab ( 2 and $10 \mathrm{mg} / \mathrm{kg}$ every 3 weeks) prolonged significantly survivals in all population (HR 0.71 and 0.61 for 2 and $10 \mathrm{mg} / \mathrm{kg}$ doses, respectively), with better results among "strongly positive" patients, whose tumors expressed PD-L1 on at least $50 \%$ of cells HR 0.54 and 0.50 for 2 and $10 \mathrm{mg} / \mathrm{kg}$ doses, respectively). Differing from other immuno-agents' trials, the population of Keynote-010 was younger, with median age of 63 years, with 429 patients (41\%) aged more than 65 years and no patients older than 70 years. Interestingly, pembrolizumab reduced the risk of death of $37 \%$ among patients younger than 65 years (HR 0.63; 95\% CI, 0.50-0.79), while a non-significant $24 \%$ reduction was observed in the 65-69 years group (HR 0.76; 95\% CI, 0.57-1.02) (22). The safety profile of pembrolizumab was consistent with nivolumab reporting fewer high-grade treatment-related AEs than docetaxel. Decrease appetite (14\%), fatigue (14\%), nausea (11\%), rash $(9 \%)$, diarrhea $(7 \%)$, and asthenia $(6 \%)$ were the most common any grade treatment-related AEs. Pembrolizumab was associated with a range of immuno-related AEs, at both doses (at $2 \mathrm{mg} / \mathrm{kg}: 20 \%$, at $10 \mathrm{mg} / \mathrm{kg}: 19 \%$ ), mostly hypothyroidism ( $8 \%$ at both doses), pneumonitis ( $5 \%$ vs. $4 \%$, respectively), and hyperthyroidism ( $4 \%$ vs. $6 \%$, respectively) (22).

Recently, data from the Keynote-024 trial moved pembrolizumab to front-line therapy in patients whose tumors expressed $\mathrm{PD}-\mathrm{L} 1 \geq 50 \%$. In the pivotal trial, 305 untreated patients were randomly assigned to pembrolizumab $(200 \mathrm{mg}$ flat dose every three weeks for up to 2 years) or platinum-based doublet chemotherapy. After a median follow up of 11.2 months, the PFS as primary end-point was significantly improved by approximately for months with pembrolizumab (10.3 vs. 6.0 months of chemotherapy, HR 0.50), and the trial was stopped early after the superior efficacy of pembrolizumab at second interim analysis (at 6 months OS rate: $80.2 \%$ vs. $72.4 \%$; HR 0.60, $\mathrm{P}=0.005)$. The improvements were seen across all analyzed subgroups, including the 164 patients aged 65 or more (54\% of enrolled population), where the HR for OS in those receiving pembrolizumab was 0.45 (23).

\section{Anti-PD-L1 agents: atezolizumab, avelumab and durvalumab}

The efficacy of anti-PDL1 agents in elderly is only preliminary, considering the lack of specific efficacy data by age and in absence of randomized phase III data, except for atezolizumab.

Atezolizumab, a type IgG1 fully humanized monoclonal antibody, blocks primarily the interaction ligand $\mathrm{PD}$ L1/receptor PD-1, and it is the last added to the list of immunotherapy drugs approved by Food and Drug Administration (FDA) in the second-line setting for NSCLC, regardless of tumor PD-L1 status. Two randomized clinical trials (OAK and POPLAR) compared atezolizumab (1,200 $\mathrm{mg}$ flat dose every 3 weeks) to docetaxel in a total of 1,137 patients with squamous or adenocarcinomatous NSCLC progressing after one or more platinum-based combination regimens $(24,25)$. In both trials, atezolizumab significantly improved OS compared to docetaxel, reporting a median survival longer than 1 year (median OS in atezolizumab arm: 12.6 and 13.8 months in POPLAR and OAK trial, respectively) and a $27 \%$ reduction in risk of death. In contrast to phase II POPLAR data reporting an absence of significant survival benefit in negative PD-L1 patients (PD-L1 expression $<1 \%$ in both tumor and immune cells), the phase III OAK trial confirmed 
the survival benefit of atezolizumab regardless of PD-L1 expression, with a significant $25 \%$ improvement in survival also among those PD-L1 negative. Regarding atezolizumab, aged-specific survival data are available for OAK trial, only. About 397 patients aged $\geq 65$ years enrolled in OAK, the risk of death was reduced of $34 \%$ with atezolizumab compared to docetaxel (HR 0.66; 95\% CI, 0.52-0.83). Recently, survival data from the cohorts of patients $65-75$ and 75 years old were presented: atezolizumab confirmed a significant survival improvement for the first subgroup (HR 0.63), with a trend toward improved survival across very elderly patients aged older than 75 years (HR 0.80) (26).

Differing to atezolizumab, the other antibody targeting PD-L1 avelumab is able to induce an antibody dependent cell-mediated cytotoxicity against tumor cells. Data from the large phase I multicohort dose-escalation and doseexpansion JAVELIN trial showed avelumab as early promise in front-line NSCLC patients who were not preselected for PD-L1 expression. Among 156 untreated patients with advanced NSCLC, avelumab (at $10 \mathrm{mg} / \mathrm{Kg}$ biweekly) had a good tolerability, with an interesting ORR of $22.4 \%$ and with a median PFS of 17.6 weeks. Notably, more than half of study population (105 patients, $67 \%$ ) was aged more than 65 years, reporting an ORR of $25 \%$, consistently to overall population (27).

Regarding the heterogeneous cohort of 184 heavily pretreated ( $33 \%$ of patients were in $\geq 3 \mathrm{rd}$ line of therapy), PD-L1 unselected [epidermal growth factor receptor (EGFR)/KRAS mutated and anaplastic lymphoma kinase (ALK) translocated patients were included] advanced NSCLC patients, avelumab demonstrated a toxicity profile and an antitumor activity similar to other immuno-agents: the ORR was $12 \%$ (recently updated to $14.1 \%$ ), and 1 -year PFS and OS rates were $18 \%$ and $36 \%$, respectively, with a median OS of 8 months (28). From exploratory analysis, a PFS benefit occurred for PD-L1 positive patients ( $\geq 1 \%$ as cutoff for tumor cell staining) relative to PD-L1 negative counterparts (12.0 vs. 5.9 months, HR 0.27-0.75), while survival curves are approached at 1 year (1-year OS: $39 \%$ vs. $36 \%$ for PD-L1 positive and negative, respectively) (28).

Two ongoing randomized phase III trials are currently comparing avelumab to first-line chemotherapy in NSCLC patients with PL-1 expression at least of $1 \%$ (JAVELIN Lung 100 phase III trial; NCT02576574), and comparing to docetaxel in the second-line setting after platinumbased doublet chemotherapy failure (JAVELIN Lung 200; NCT02395172).

Durvalumab is another IgG1 human antibody that blocks
PD-L1 binding to PD-1 and B7-1, but it does not affect the interaction between PD-1 and PD-L2, probably avoiding immune toxicities associated with that interference. Durvalumab showed promising activity as a first- or subsequent- line of treatment in 304 patients enrolled in a phase I/II study. A total of 50 confirmed responses was reported, with higher response rates among patients with high PD-L1 expression, defined as at least $25 \%$ of tumor cells staining for PD-L1 (ORR: 25\% vs. 6\% in high and low PD-L1 expression, respectively). Interestingly, more than half of pretreated patients with high PD-L1 expression were alive at 1 year receiving durvalumab as second-line (56\%) as well as third-line treatment (51\%) (29).

Based on these data, durvalumab was investigated as third-line or higher advanced NSCLC in the phase II ATLANTIC trial. The accrual initially was irrespective of PD-L1 status and later it was restricted to higher PD-L1 expression level, with $25 \%$ as cutoff. Data from more than 300 EGFR wild-type and ALK not-rearranged, heavilypretreated, NSCLC patients confirmed response rates to durvalumab proportionally with $\mathrm{PD}-\mathrm{L} 1$ expression levels, with ORR of $16.4 \%$ and $30.9 \%$ in patients with PD-L1 $\geq 25 \%$ and $\geq 90 \%$, respectively enrolled in cohort 2 and 3 (ORR PD-L1 low/negative: 7.6\%). Similarly, 1-year OS rates were $47 \%$ for PD-L $1 \geq 25 \%$ and $50.8 \%$ for PD-L 1 $\geq 90 \%$. Out of the 101 patients older than 65 years, the ORR was $20 \%$ in PD-L1 $\geq 25 \%$ population and $3 \%$ in PD$\mathrm{L} 1<25 \%$, in contrast to $13 \%$ and $11 \%$ reported in the younger population, respectively. These data suggest the potential role of age on immune response in those patients with lower PD-L1 expression levels (30).

\section{Conclusions}

If, or how much, age plays a role in immune checkpoint inhibitor response or toxicity is still a matter of debate, representing a relevant unmet need. Efficacy and safety data by age in the phase III trials leading to FDA approval of immune checkpoint inhibitors are limited, with a modest representation of older adults on these pivotal trials. However, further assessment in the elderly is warranted, considering the high prevalence of lung cancer among these setting of patients.

Furthermore, the immuno-agents appear generally well tolerated, with lower rates of severe AEs (G3-4 AEs: 7-13\%) although details of AEs by age have not been reported thus far.

In absence of any randomized trials designed specifically for the elderly population and in the highly complex 
scenario of divergent clinical trials, data from three large alternative studies (EAP and retrospective cohorts studies) showed comparable survival outcomes in patients aged 70 or more compared to younger ones. Further research is needed to evaluate the effectiveness and the toxicity of immuneagents in this growing population. The better knowledge of aging immune system changes and their interaction with immune checkpoint inhibitors will be helpful to guide the management of immunotherapies in elderly.

\section{Acknowledgements}

None.

\section{Footnote}

Conflicts of Interest: C Gridelli received honoraria as speaker bureau and advisory board for Astra Zeneca, BMS, MSD, Roche. The other authors have no conflicts of interest to declare.

\section{References}

1. Owonikoko TK, Ragin CC, Belani CP, et al. Lung cancer in elderly patients: an analysis of the surveillance, epidemiology, and end results database. J Clin Oncol 2007;25:5570-7.

2. Balducci L, Extermann M. Management of cancer in the older person: a practical approach. Oncologist 2000;5:224-37.

3. Gridelli C, Aapro M, Ardizzoni A, et al. Treatment of Advanced Non-Small-Cell Lung Cancer in the Elderly: Results of an International Expert Panel. J Clin Oncol 2005;23:3125-37.

4. Fulop T, Kotb R, Fortin CF, et al. Potential role of immunosenescence in cancer development. Ann N Y Acad Sci 2010;1197:158-65.

5. Ferrara R, Mezquita L, Auclin E, et al. Immunosenescence and immunecheckpoint inhibitors in non-small cell lung cancer patients: Does age really matter? Cancer Treat Rev 2017;60:60-8.

6. Champiat S, Dercle L, Ammari S, et al. Hyperprogressive Disease Is a New Pattern of Progression in Cancer Patients Treated by Anti-PD-1/PD-L1. Clin Cancer Res 2017;23:1920-8.

7. Chen DS, Mellman I. Oncology meets immunology: the cancer-immunity cycle. Immunity 2013;39:1-10.

8. Chen YM. Immune checkpoint inhibitors for nonsmall cell lung cancer treatment. J Chin Med Assoc. 2017;80:7-14.

9. Brahmer J, Horn L, Jackman D, et al. Five-year followup from the CA209-003 study of nivolumab in previously treated advanced non-small cell lung cancer (NSCLC): Clinical characteristics of long-term survivors. 2017 AACR Annual Meeting. Abstract CT077. Presented April 3, 2017.

10. Gettinger SN, Horn L, Gandhi L, et al. Overall Survival and Long-Term Safety of Nivolumab (Anti-Programmed Death 1 Antibody, BMS-936558, ONO-4538) in Patients With Previously Treated Advanced Non-Small-Cell Lung Cancer. J Clin Oncol 2015;33:2004-12.

11. Rizvi NA, Mazières J, Planchard D, et al. Activity and safety of nivolumab, an anti-PD-1 immune checkpoint inhibitor, for patients with advanced, refractory squamous non-small-cell lung cancer (CheckMate 063): a phase 2, single-arm trial. Lancet Oncol 2015;16:257-65.

12. Lena H, Rizvi NA, Wolf J, et al. 137O: Nivolumab in patients (pts) with advanced refractory squamous (SQ) non-small cell lung cancer (NSCLC): 2-year follow-up from CheckMate 063 and exploratory cytokine profling analyses. J Thorac Oncol 2016;11:S115-6.

13. Brahmer J, Reckamp KL, Baas P, et al. Nivolumab versus Docetaxel in Advanced Squamous-Cell Non-Small-Cell Lung Cancer. N Engl J Med 2015;373:123-35.

14. Borghaei H, Paz-Ares L, Horn L, et al. Nivolumab versus Docetaxel in Advanced Nonsquamous Non-Small-Cell Lung Cancer. N Engl J Med 2015;373:1627-39.

15. Font EF, Gettinger SN, Burgio MA, et al. Three-year follow-up from CheckMate 017/057: Nivolumab versus docetaxel in patients with previously treated advanced non-small cell lung cancer (NSCLC). Ann Oncol 2017;28:460-96.

16. Carbone DP, Reck M, Paz-Ares L, et al. First-Line Nivolumab in Stage IV or Recurrent Non-Small-Cell Lung Cancer. N Engl J Med. 2017;376:2415-26.

17. Gettinger SN, Horn L, Ramalingam SS, et al. 3094 Nivolumab (NIVO) safety profile: Summary of findings from trials in patients (pts) with advanced squamous (SQ) non-small cell lung cancer (NSCLC). Eur J Cancer 2015;51:S631.

18. Spigel D, Schwartzberg L, Waterhouse D, et al. P3.02c-026 Is Nivolumab Safe and Effective in Elderly and PS2 Patients with Non-Small Cell Lung Cancer (NSCLC)? Results of CheckMate 153. J Thorac Oncol 2017;12:S1287.

19. Bagley S, Kothari S, Aggarwal C, et al. P3.02c-028 outcomes of nivolumab in elderly patients (pts) with non-small cell lung cancer (NSCLC). J Thorac Oncol 


$$
\text { 2017;12:S1289. }
$$

20. Grossi F, Crinò L, Catino A, et al. P3.02c-096 Use of Nivolumab in Elderly Patients with Advanced Squamous NSCLC: Results from the Italian Expanded Access Programme (EAP). J Thorac Oncol 2017;12:S1337-8.

21. Migliorino MR, Gelibter A, Grossi F, et al. 1320P Use of nivolumab in elderly patients with advanced nonsquamous NSCLC: Results from the Italian expanded access program (EAP). Ann Oncol 2017;28:v460-96.

22. Herbst RS, Baas P, Kim DW, et al: Pembrolizumab versus docetaxel for previously treated, PD-L1-positive, advanced non-small-cell lung cancer (KEYNOTE-010): A randomized controlled trial. Lancet 2016;387:1540-50.

23. Reck M, Rodríguez-Abreu D, Robinson AG, et al. Pembrolizumab versus chemotherapy for PD-L1positive non-small-cell lung cancer. N Engl J Med 2016;375:1823-33.

24. Rittmeyer A, Barlesi F, Waterkamp D, et al. Atezolizumab versus docetaxel in patients with previously treated non-small cell lung cancer (OAK): a phase 3, openlabel, multicentre randomized controlled trial. Lancet 2017;389:255-65.

25. Fehrenbacher L, Spira A, Ballinger M, et al. Atezolizumab versus docetaxel for patients with previously treated nonsmall-cell lung cancer (POPLAR): a multicentre,

Cite this article as: Casaluce F, Sgambato A, Maione P, Spagnuolo A, Gridelli C. Lung cancer, elderly and immune checkpoint inhibitors. J Thorac Dis 2018;10(Suppl 13):S1474S1481. doi: $10.21037 /$ jtd.2018.05.90 open-label, phase 2 randomised controlled trial. Lancet 2016;387:1837-46.

26. Gadgeel S, Ciardiello F, Rittmeyer A, et al. PL04a.02: OAK, a randomized $\mathrm{Ph}$ III study of atezolizumab vs docetaxel in patients with advanced NSCLC: results from subgroup analyses. J Thorac Oncol 2017;12:S9-10.

27. Jerusalem G, Chen FL, Spigel D, et al. OA03.03 JAVELIN Solid Tumor: Safety and clinical activity of avelumab (antiPD-L1) as first-line treatment in patients with advanced NSCLC. J Thorac Oncol 2017;12:S252.

28. Gulley JL, Rajan A, Spigel DR, et al. Avelumab for patients with previously treated metastatic or recurrent non-smallcell lung cancer (JAVELIN Solid Tumor): dose-expansion cohort of a multicenter, open-label, phase $1 \mathrm{~b}$ trial. Lancet Oncol 2017;18:599-610.

29. Antonia SJ, Brahmer JR, Khleif S, et al. Phase 1/2 study of the safety and clinical activity of durvalumab in patients with non-small cell lung cancer (NSCLC). Presented at European Society for Medical Oncology 2016 Congress, 1216PD. Annals of Oncology 2016;27:416-54.

30. Garassino M, Vansteenkiste J, Kim JH, et al. PL04a.03: Durvalumab in $\geq 3$ rd-line locally advanced or metastatic, EGFR/ALK Wild-Type NSCLC: results from the Phase 2 ATLANTIC study. J Thorac Oncol 2017;12:S10-1. 